\title{
A constraint-driven approach to food supply chain management
}

\author{
Pawel Sitek and Jaroslaw Wikarek \\ Department of Information Systems, Kielce University of Technology, \\ Kielce, Poland, and \\ Peter Nielsen \\ Department of Mechanical and Manufacturing Engineering, Aalborg University, \\ Aalborg, Denmark
}

\begin{abstract}
Purpose - The purpose of this paper is the need to build a novel approach that would allow flexible modeling and solving of food supply chain management (FSCM) problems. The models developed would use the data (data-driven modeling) as early as possible at the modeling phase, which would lead to a better and more realistic representation of the problems being modeled.

Design/methodology/approach - An essential feature of the presented approach is its declarativeness. The use of a declarative approach that additionally includes constraint satisfaction problems and provides an opportunity of fast and easy modeling of constrains different in type and character. Implementation of the proposed approach was performed with the use of an original hybrid method in which constraint logic programming (CLP) and mathematical programming (MP) are integrated and transformation of a model is used as a presolving technique.

Findings - The proposed constraint-driven approach has proved to be extremely flexible and efficient. The findings obtained during part of experiments dedicated to efficiency were very interesting. The use of the constraint-driven approach has enabled finding a solution depending on the instance data up to 1,000 times faster than using the MP.

Research limitations/implications - Due to the limited use of exact methods for NP-hard problems, the future study should be to integrate the CLP with environments other than the MP. It is also possible, e.g., with metaheuristics like genetic algorithms, ant colony optimization, etc.

Practical implications - There is a possibility of using the approach as a basis to build a decision support system for FSCM, simple integration with databases, enterprise resource planning systems, management information systems, etc.

Originality/value - The new constraint-driven approach to FSCM has been proposed. The proposed approach is an extension of the hybrid approach. Also, a new decision-making model of distribution and logistics for the food supply chain is built. A presolving technique for this model has been presented.
\end{abstract}

Keywords Mathematical programming, Decision support, Food supply chain management,

Constraint logic programming, Constraint satisfaction problem, Data-driven computing

Paper type Research paper

\section{Introduction}

The sets of processes, operations and facilities that assist in changing the food from its raw material state to our plates is known as the food supply chain (FSC). The supply chain (SC) starts with a producer (farmer, agriculture organization, food manufacturing) and, at this stage, moves through various methods of food processing and manufacturing. Between each stage, the movement of material is facilitated by transportation and logistics companies. Most often, these are third party logistics and fourth party logistics companies. The companies ensure that the food products reach customers (retailers, end users, etc.) on

(C) Pawel Sitek, Jaroslaw Wikarek and Peter Nielsen. Published by Emerald Publishing Limited. This article is published under the Creative Commons Attribution (CC BY 4.0) licence. Anyone may reproduce, distribute, translate and create derivative works of this article (for both commercial \& non-commercial purposes), subject to full attribution to the original publication and authors. The full terms of this licence may be seen at http://creativecommons.org/licences/by/4.0/legalcode
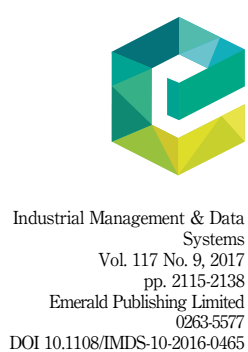
IMDS 117,9

2116

time and have proper quality. In other words, every step of the food industry's chain of production and distribution is regulated "from farm to fork" so to speak.

The food sector is a very complex environment influenced by technological, social, economic, industrial, legal, business and other factors that shape the level of the availability of food, the nature of the food product and the delivery of the food products. The complexity and complications in the FSC derive from many areas: consumer and market choices, agriculture and food production, manufacturing, processing and maintaining quality, distribution and logistics, etc. Different actors within the FSC aim to increase efficiency and improve the performance of the SC in terms of quantity, speed, quality and pricing, while maintaining the necessary requirements for food product safety (Banaeian et al., 2015). The actors and their places in FSC and the basic structure of FSC are shown in Figure 1. The literature offers numerous models and solution methods for food supply chain management (FSCM) problems (Section 2). In all these models, areas and components of FSCs, a certain set of constraints can be identified and described. The most important constraints are precedence constraints, those related to production and distribution capacity, time constraints, environmental constraints and those related to perishability, shelf times, traceability, strong seasonal behavior, temperature evolution during transportation, traveling time, etc. Effective modeling and management of the whole SC will thus be a key issue to accomplish. Finding such an effective modeling and management method was the motivation of this research. As a result, a constraint-driven approach to FSCM is proposed, based on declarative environments supported by constraint satisfaction problem (CSPs) (Rossi et al., 2006) and constraint logic programming (CLP) (Apt and Wallace, 2006) (Section 3). This constraint-driven approach is a generalization of data-driven control, which was earlier used in parallel programming.

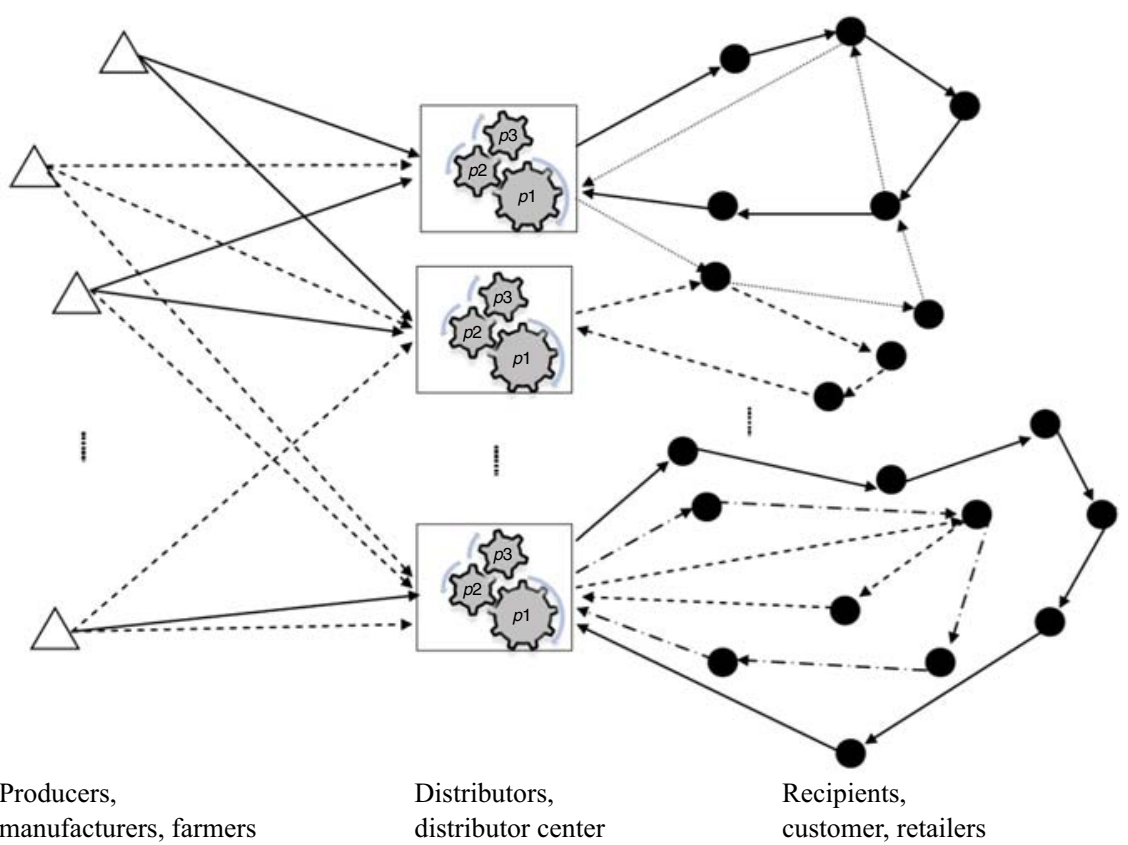

Figure 1.

The food supply chain network manufacturers, farmers

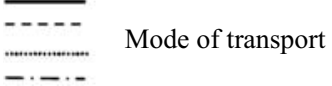

distributor center

Recipients,

customer, retailers 
Data-driven methods find application in modeling, optimization, decision support as well as in the analysis of big data sets (Kang et al., 2014; Power, 2008; Kutz, 2013).

The new constraint-driven approach proposes a new flexible and dedicated method for modeling of problems based on the collections of facts, questions and constraints, characterized by greater search efficiency. Modeling flexibility is the ability to model any type of constraints. Model dedication results from the use of specific data instances as early as possibleat the modeling phase. Efficiency is a result of the properties of the constraint-based methods and, above all, of the proposed transformation of the model, which is the integral part of the constraint-based approach.

To illustrate the proposed approach, a logistics and distribution model for FSCM was developed in the form of a set of CSPs, a set of management questions and a set of facts (Section 4). Due to its ability to implement the set of various management questions, the model is a suitable tool for management support in both areas. The data layer of the model, in the form of a set of facts, can be easily integrated with relational data bases, data warehouses and even XML-type flat files. The implementation of the proposed solutions was performed with the use of the original hybrid approach (Sitek and Wikarek, 2013, 2016; Sitek, 2014). The results are shown in Section 4. The summary of the further research and development options for the presented approach is shown in Section 5.

\section{FSCM - background and models}

Cost reduction and increasing responsiveness are two main traditional concerns in SCs. Through the supply chain management (SCM), it aims to achieve better customer service with less cost while satisfying various requirements and different types of constraints for all participants in the entire chain (Van der Vorst et al., 2005). Nowadays, in the food sector, the consumers demand high-quality food with various novel forms throughout the year with a good and competitive price (Trienekens and Zuurbier, 2008; Nakandala et al., 2016). Thus, food quality and waste issues are two main differences between SCM and FSCM. The FSC issues are ongoingly under public scrutiny and investigation. This is the result of several factors, such as food-related diseases, e.g. BSE, EHEC, etc., distribution practices used to bring the food products into shelves of the nearest market, globalization, changes in eating habits, having accurate information on the ingredients and the expiry date, etc. Thereby, a very important element in the FSC is traceability. In this context, it is very important to achieve end-to-end traceability across the chain. This is currently quite a challenge from a technical, coordination and cost perspective.

Kelepouris et al. (2007) show that the RFID technology can meet traceability requirements and discuss which technological approach is more appropriate in a given context.

Taking also into account the previously mentioned perishability, shelf times, traceability, strong seasonal behavior, etc., modern FSCM is a very complex problem. This complexity is especially critical when dealing with perishable food products where the delivery/traveling time through the entire $\mathrm{SC}$ and the opportunities to use distribution centers and warehouses as buffers are constrained. The complexity increases even further if one takes into account the sustainability (Linton et al., 2007). Sustainable development in this area deals with balances between ecological, economic, social behaviors and eating habits. Therefore, the quality of the product that is delivered by the producers and distributors to a customer needs to be environmentally safe, competitive, socially fair and profitable (Kepler, 2004). All this results in the need to seek innovative and advanced models and approaches to FSCM. In the reviewed literature (Banaeian et al., in press; Saha et al., 2017; Soysal et al., 2012; Ahumada and Villalobos, 2009; Soto-Silva et al, 2016), researchers and practitioners have proposed various types of models. The most common are: mixed integer programming, linear programming (LP), mixed integer linear programming, meta-heuristics, multi-objective programming, stochastic programming, fuzzy logic (Bocewicz et al., 2016), simulation or analytical models, etc. 
IMDS

117,9

2118

Depending on the complexity of the problem in some implementation in addition to exact approaches derived from operational research (OR) (Schrijver, 1998), the authors develop several heuristics to search for near-optimal solutions (Brown et al., 2001; Osvald and Stirn, 2008; Wang et al., 2010). In addition, the analyzed models (Soysal et al., 2012; Soto-Silva $e$ t al., 2016) usually include only selected limited aspects and areas of the FSCM evaluated according to various performance indicators (e.g. various SC costs, utilization of transport carriers, quality decay tracking, etc.).

The application of the classical approaches based on OR methods to FSCM problems has multiple shortcomings. The most critical of them are:

- Easy modeling refers only to linear and integer constraints, including binary constraints. In FSC, non-linear constraints and fuzzy cost functions are often encountered.

- The effectiveness of finding solutions drops substantially with the number of integer and binary decision variables. When modeling FSCs, the scale of the formulated problem is usually very large, which prescribes either heuristic methods or problem decomposition.

- The model is completely separated from data. FSCs, especially fresh FSCs, are in a constant state of change, which makes working on static data counterproductive.

- Partial modification of the model is difficult, e.g. when new constraints are added or new evaluation criteria are used (in this case, the model usually has to be built from scratch), FSCs dynamically add/remove members and products as seasons change, etc.

- The variable domains cannot be used in the solution process.

Considering the above reasons and the specific nature of FSCM problems (a large number of different types of constraints), the main motivation of this study is the need for a novel approach that, on the one hand, would allow flexible modeling and solving of FSCM problems and, on the other hand, use the data (data-driven modeling) as early as possible at the modeling phase. This would lead to a better and more realistic representation of the problems being modeled. Constraint-based environments (Rossi et al., 2006) have all these attributes. These environments are declarative (and therefore easily generalized and updated) and supported by a possibility of solving CSPs. The main contribution of the present work is to propose and implement a constraint-driven approach to FSCM. This approach allows modeling through the constraints included in the problems. It is declarative and therefore asking questions is easy, and the data layer is based on facts, which makes up for an incredibly flexible solution, easy to integrate with various data sources and systems.

\section{A constraint-driven approach - background}

Constructing a uniform approach to both modeling and solving decision problems within FSCM is the key issue. This results from the multiplicity and diversity of the models and methods to solve them which makes FSCM very complex. Changing the objective function, and modifying or adding constraints, frequently in the FSC results in the need for a profound modification or construction of new models. This applies to OR-based approaches, simulations or heuristics. In contrast, the proposed uniform constraint-driven approach is based on the declarative programming paradigm and supplemented with constraint solving methods. In the declarative approach, stating and describing the problem represent its model and solution. Adding new constraints is easy. Declarative programming is a host that includes a number of better-known programming paradigms. One of these is CLP, where the classical declarative approach of logic programming is complemented with a possibility of solving CSPs. A CSP is a mathematical problem defined as a set of objects whose state must satisfy a number of constraints (Banaszak et al., 2009). The CSP represents the objects in a 
problem as a set of finite constraints over decision variables, which is solved by constraint satisfaction methods. Formally, a CSP is defined as a triple (Var, Dom, Con), where Var $=\left\{\operatorname{Var}^{1}, \operatorname{Var}^{2}, \ldots, \operatorname{Var}^{n}\right\}$ is a set of decision variables, Dom $=\left\{\operatorname{Dom}^{1}, \operatorname{Dom}^{2}, \ldots, \operatorname{Dom}^{n}\right\}$ is a set of the corresponding domains of values and $\operatorname{Con}=\left\{\operatorname{Con}^{1}, \operatorname{Con}^{2}, \ldots, \mathrm{Con}^{m}\right\}$ is a set of constraints binding decision variables. An evaluation of the decision variables is a function from a subset of variables to a particular set of values in the respective subset of domains. An evaluation is consistent if it does not violate any of the constraints form the set Con. An evaluation is complete if it includes all decision variables from the set $V a r$. An evaluation is a solution if it is consistent and complete; such an evaluation is said to solve the CSP. CSPs on finite domains are usually solved using a different form of search techniques and methods. The most used techniques are variants of constraint propagation, local search, and backtracking. For example, a CLP can use artificial intelligence techniques to improve the search: constraint propagation, data-driven computation, look ahead and forward checking. But the most important key innovation behind CLP is constraint propagation. Propagation is a generalization of data-driven computation. Much of the power of CLP derives from the data-driven way in which the consequences of changes are propagated through a constraint network. It can therefore be concluded that the constraint-driven behavior is a generalization and extension of data-driven control (Schimpf and Kish, 2012) Unfortunately, for linear constraints with multiple variables and optimization problems, CLP performs much worse. Mathematical programming (MP)-based environments perform much better in those areas. Therefore, the literature (Akkerman et al., 2010; Milano and Wallace, 2010; Achterberg et al., 2008; Bockmayr and Kasper, 2004) and our own studies (Sitek, 2014; Sitek and Wikarek, 2016) have focused on the integration of CLP and MP to utilize their respective strengths and make up for their shortcomings.

\subsection{A constraint-driven approach - concept and assumptions}

The main assumptions adopted for the construction of the constraint-driven approach are as follows:

- CLP is the primary environment to embed the approach;

- the data layer comprises a set of facts - for the illustrative example, as in Figure 6;

- the constraints contained in the problem are grouped and form adequate sets of CSPs;

- the criteria of decision/solution optimality are modeled with the use of $w h$-questions, e.g., what is the maximum [...], what is the minimum [...], etc.;

- feasibility criteria for the decision/solution are modeled with the aid of general questions, e.g., is it possible [...]? Is it possible to realize [...]? Is it possible to perform $[\ldots]$ ? etc.;

- the model of the problem comprises a set of CSPs, a set of questions and a set of facts (Figure 4), and is implemented in the form of sets of predicates and facts;

- extension and/or modification of the model involves adding new predicates, fact instances or new facts;

- the questions can refer to the entire set of CSPs or its parts;

- the model is subject to presolving through transformation; and

- implementation was performed with the aid of the hybrid approach that integrates CLP and MP environments.

Figure 2 shows a general diagram of the functional concept of the constraint-driven approach. The proposed approach consists of the following phases: modeling, 


\section{IMDS \\ 117,9}

Figure 2.

The concept diagram of constraint-driven approach

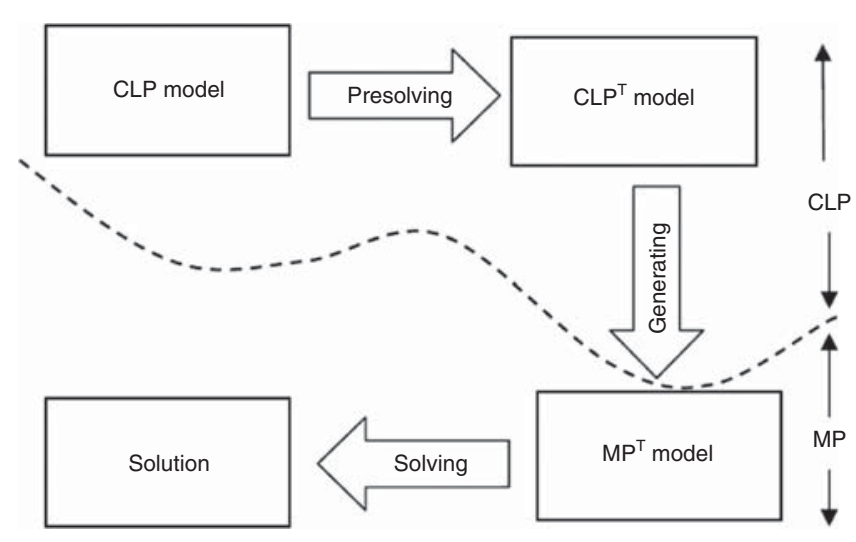

implementation, presolving, generation and solving. Except for the last phase, implemented in MP, all the phases are completed in the CLP environment. A functional diagram showing the operation mode of the proposed constraint-driven approach is presented in Figure 3.

First, a CLP model is built according to the concept as in Figure 4. Its implementation is performed through a set of predicates and facts. Then the CLP is presolved with the aid of model transformation and constraint propagation. This is an original method designed by the authors of this paper (Sitek and Wikarek, 2015, 2016). The method, based on data instances and incorporated CLP techniques, allows the transformation of decision variables (their aggregation and reduction) and, analogously, constraints. After the transformation, the CLP ${ }^{\mathrm{T}}$ model is obtained. At the same time, the domain solution is obtained. It includes the values of all domains of the transformed decision variables that meet a given set of constraints. This model and the domain solution are the basis for generating, with adequate predicates, the final model as an $\mathrm{MP}^{\mathrm{T}}$ model that is solved by the MP solver.

\section{Illustrative example for a constraint-driven approach}

The authors' model for the management of logistics and distribution for FSC was presented as an illustrative example for a constraint-driven approach. The model was formulated as a set of $\mathrm{CSP}_{1}, \mathrm{CSP}_{2}, \mathrm{CSP}_{3}$ and $\mathrm{CSP}_{\mathrm{TS}}$, set of facts (Figure 5) and a set of questions (Table II). The problem description for illustrative example is presented in Section 4.1. The formalization of CSPs, questions and the structure of the facts for illustrative example are proposed in Section 4.2. Presolving techniques for the illustrative model are discussed in Section 4.3. In the end, calculation examples are presented in Section 4.4.

\subsection{Illustrative example - problem description}

Given is a distribution system for FSCM, defined by a set of producers (farmers, processors, manufacturing plants, etc.) $K=\left\{k_{1}, k_{2}, \ldots, k_{i}, \ldots, k_{L K}\right\}$, where $L K$ is the number of producers. The producers supply/produce a specific set of products $P=\left\{p_{1}, p_{2}, \ldots, p_{i}, \ldots, p_{L P}\right\}$, where $L P$ is the number of products and $V P_{p}$ factor defines the product dimension/size $p(p \in P)$. The $\mathrm{CK}_{k, p}$ factor specifies the quantity of product $p(p \in P)$ the producer $k(k \in K)$ is able to supply, and the $W_{k, p}$ factor gives the cost of product $p(p \in P)$ production incurred by producer $k(k \in K)$. Products $p$ are categorized. The set of all categories is denoted by $G=\left\{g_{1}, g_{2}, \ldots, g_{i}, \ldots, g_{L G}\right\}$, where $L G$ is the number of categories. A given product can be assigned to only one category (factor $A_{p, g}=1$ means that product $p(p \in P)$ is in category 


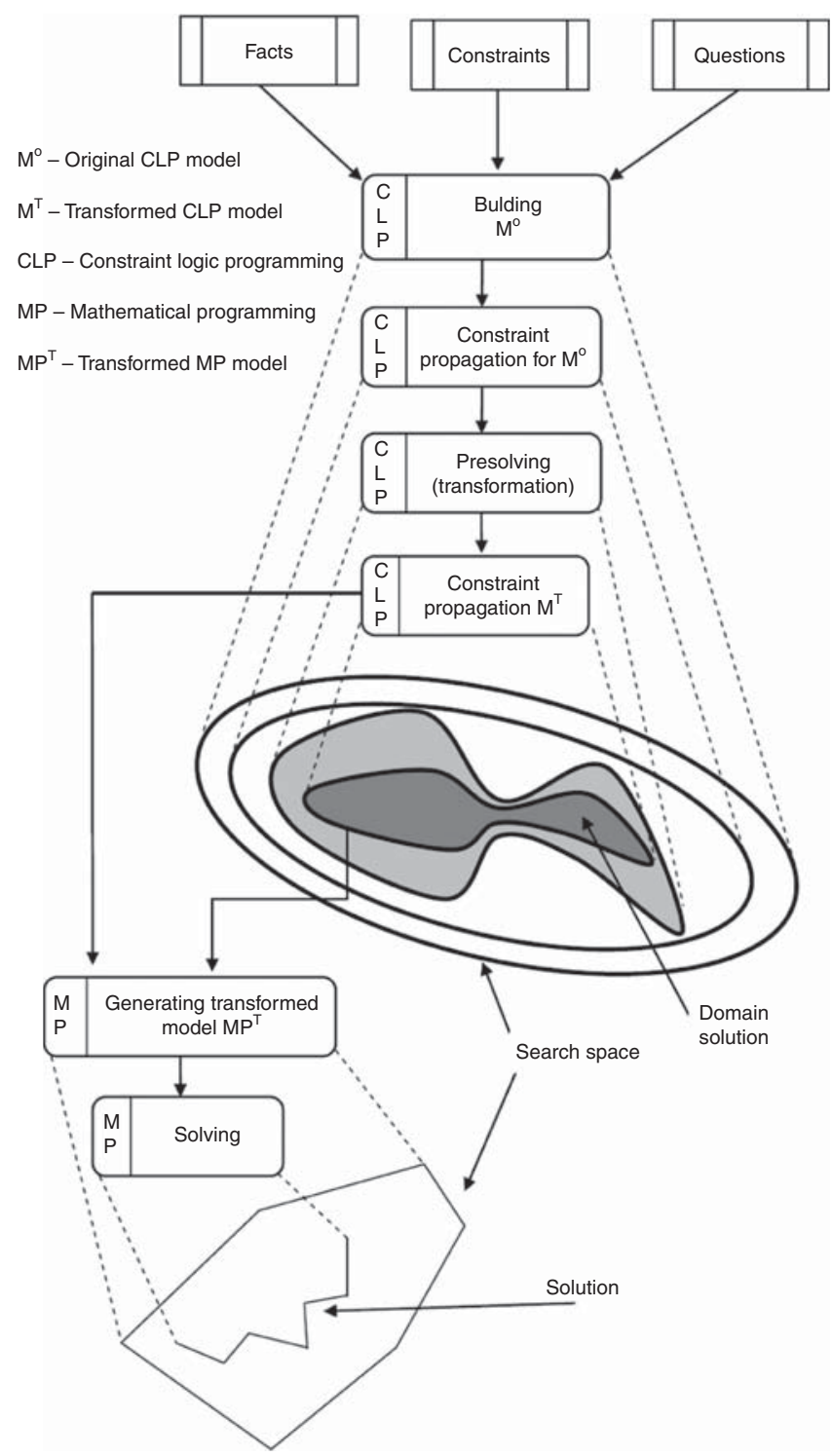

Figure 3.

The functional diagram of constraintdriven approach

$g(g \in G)$, otherwise $\left.A_{p, g}=0\right)$. The products are ordered by specified customers $O=\left\{o_{1}, o_{2}\right.$, $\left.\ldots, o_{i}, \ldots, o_{L O}\right\}$, where $L O$ is the number of customers. The factor $Z_{o, p}$ defines the quantity of product $p(p \in P)$ ordered by customer $o(o \in O)$, whereas $T O_{p, o}$ is the maximum time allowed for order execution. Customer orders should be executed in the following way: the products ordered (by different customers) are collected and transported to distribution centers $C=\left\{c_{1}\right.$, $\left.c_{2}, \ldots, c_{i}, \ldots, c_{\mathrm{LC}}\right\}$, where $L C$ is the number of centers. The factor $L_{c, g}=1$ means that the center $c(c \in C)$ is able to reload products type/category $g(g \in G)$. In the centers, the products ordered by a given customer are combined and usually placed on pallets. Then they are shipped to the customer (factor $T C_{c}$ defines the time needed to prepare the shipment by the 
IMDS

117,9

2122

Figure 4.

The model

structure for constraint-driven approach

Figure 5.

The model

structure for

illustrative example
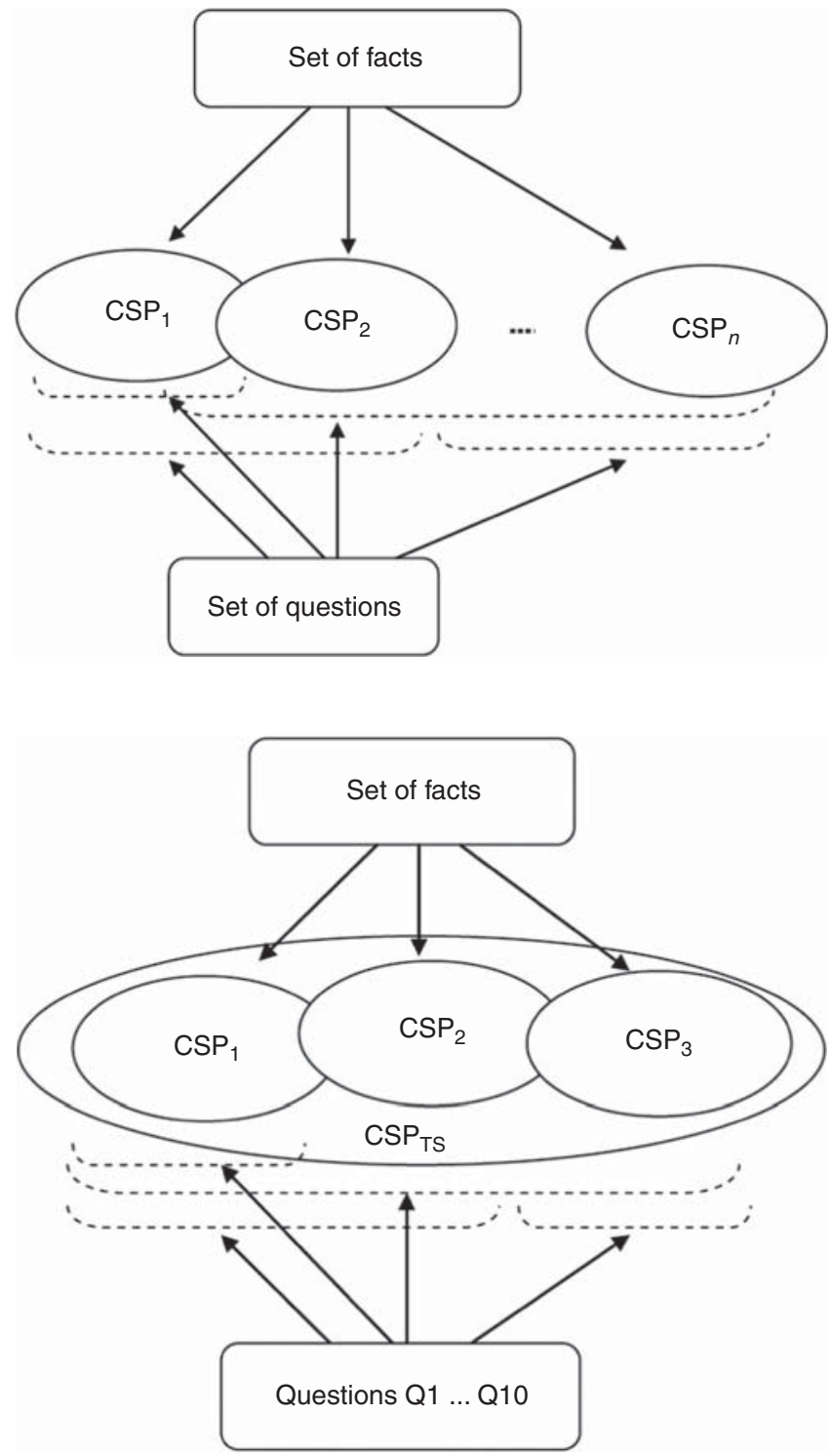

center $c(c \in C))$. One center can handle multiple customers, and a customer can be supplied from multiple centers. The factor $B_{c, o}=1$ means that the center $c(c \in C)$ can handle customer $o(o \in O)$. The delivery can be executed directly from the center to the customer or through the center - customer1-customer2, etc. - system. Deliveries are realized by specified modes of transport on the route from producer to customer $S=\left\{s_{1}, s_{2}, \ldots, s_{i}, \ldots, s_{L S}\right\}$, where $L S$ is the number of transport modes (and factor $V S_{S}$ defines the dimension of the modes $s(s \in S), K S_{S}$ defines the cost of the mode per distance unit and along the center-customer route by the mode of transport $F=\left\{f_{1}, f_{2}, \ldots, f_{i}, \ldots, f_{L F}\right\}$, where $L F$ is the number of transport modes (and factor $V F_{s}$ defines the dimension/size (capacity, payload) of the transport mode 
$f(f \in F)$. The cost of using the transport means for the distance unit is $K F_{f}$, and factor $C F_{c, f}=1$ means that the mode of transport $f(f \in F)$ can travel from the center (is assigned to the center) $c(c \in C)$. Different non-universal modes of transport are used. Each mode of transport is a specific type. If factor $H S_{s, u}=1$, the mode of transport $s(s \in s)$ belongs to type $u$ $(u \in U)$, otherwise $\mathrm{HS}_{s, u}=0$. Likewise, if factor $\mathrm{HF}_{f, u}=1$, the mode of transport $f(f \in F)$ belongs to type $u(u \in U)$. The modes of a given type can carry the products of specific categories, and factor $D_{u, g}=1$ means that mode type $u(u \in U)$ can carry products in category $g(g \in G)$, otherwise $D_{u, g}=0$. The factor $O D_{i, j}$ defines the distance from point $i(i \in K \cup C \cup O)$ to point $j(j \in K \cup C \cup O)$, and the factor $D O_{i, j}$ is the travel time from point $i(i \in K \cup C \cup O)$ to the point $j(j \in K \cup C \cup O)$.

\subsection{Illustrative example - model of the problem}

The basic element of the model (Figure 5) is a set of CSPs for different functional areas of FSCM. The set of CSPs consists of the following elements:

- $\mathrm{CSP}_{1}$ - deliveries from producers, manufacturers to distribution centers according to customer orders;

- $\mathrm{CSP}_{2}$ - preparation (picking, packing, palletization, etc.) and dispatch shipments to customers in the distribution center;

- $\mathrm{CSP}_{3}$ - deliveries from distribution centers to customers; and

- $\mathrm{CSP}_{\mathrm{TS}}$ - the set of time constraints for the delivery.

Formalization of the model as a set of CSPs is presented in Table I. A detailed description of decision variables, parameters and constraints for each CSP are, respectively, shown in Tables AI-AIII. The $\mathrm{CSP}_{\mathrm{TS}}$ for time constraints that are so important and very characteristic for FSCM, which links all CSPs, is shown in Table AIV.

The set of facts is the data layer of the model. Every fact consists of attributes. Some of them are key attributes whose values identify an instance of the fact. Others are descriptive attributes, data and so on. The structure of the facts and their relationships for illustrative model are shown in Figure 6. A description of all the attributes of the facts is presented in Appendix 2 and Section 4.1. The set of facts is due to its construction easy to integrate with other data systems like relation databases, data warehouses, XML files, etc. Therefore, the proposed model is easily inerrable with enterprise resource planning, material requirement planning, distribution requirement planning and other management information systems.

The final element of the model is the set of management questions which model specific decisions and/or quantitative evaluation of these decisions. A sample set of management questions for the illustrative model is shown in Table II. Questions Q1-Q7 are specific questions that model the optimal decisions. Questions Q8 and Q9 are general questions. These questions determine whether the decisions are allowed. The most interesting question is Q10 as it contains a logical condition, for whose implementation it

\begin{tabular}{llr}
\hline $\mathrm{CSP}$ & \multicolumn{1}{c}{ Description } & \\
\hline $\mathrm{CSP}_{1}$ & $\left.\mathrm{CSP}_{1}=(C=\{C 1, C 2, C 3, C 4, C 5, C 6)\}, X=\left\{X_{k, p, c, o, s}, Y_{k, c, s}\right\}, D=\left\{D_{X}^{1}, D_{Y}^{1}\right\}\right)$ \\
$\mathrm{CSP}_{2}$ & $\left.\mathrm{CSP}_{2}=(C=\{C 7, C 8, C 9, C 10, C 11, C 12, C 13, C 14)\}, X=\left\{X_{k, p, c, o,}, U O_{c, o, g},\right\}, D=\left\{D_{X}^{2}, D_{U O}^{2},\right\}\right)$ & Table I. \\
$\mathrm{CSP}_{3}$ & $\mathrm{CSP}_{3}=(C=\{C 15, C 16, C 17, C 18, C 19, C 20, C 21, C 22, C 23, C 24, C 25, C 26)\}$, & CSPs for \\
& $\left.X=\left\{X H_{f, i, j}, Y H_{f, i, j, h}, F H_{h, f}\right\}, D=\left\{D_{X H}^{3}, D_{Y H}^{3}, D_{F H}^{3}\right\}\right)$ & illustrative example \\
$\mathrm{CSP}_{\mathrm{TC}}$ & $\left.\mathrm{CSP}=(C=\{C 27)\}, X=\left\{X_{k, p, c, 0, s}, X O_{k, p, c, o, s}, X H_{f, i, j}, T H_{f, o}\right\}, D=\left\{D_{X}^{4}, D_{X O}^{4}, D_{X H}^{4}, D_{T H}^{4}\right\}\right)$ & \\
\hline
\end{tabular}




\section{IMDS 117,9}

Figure 6.

The structure and relationships between facts for illustrative example

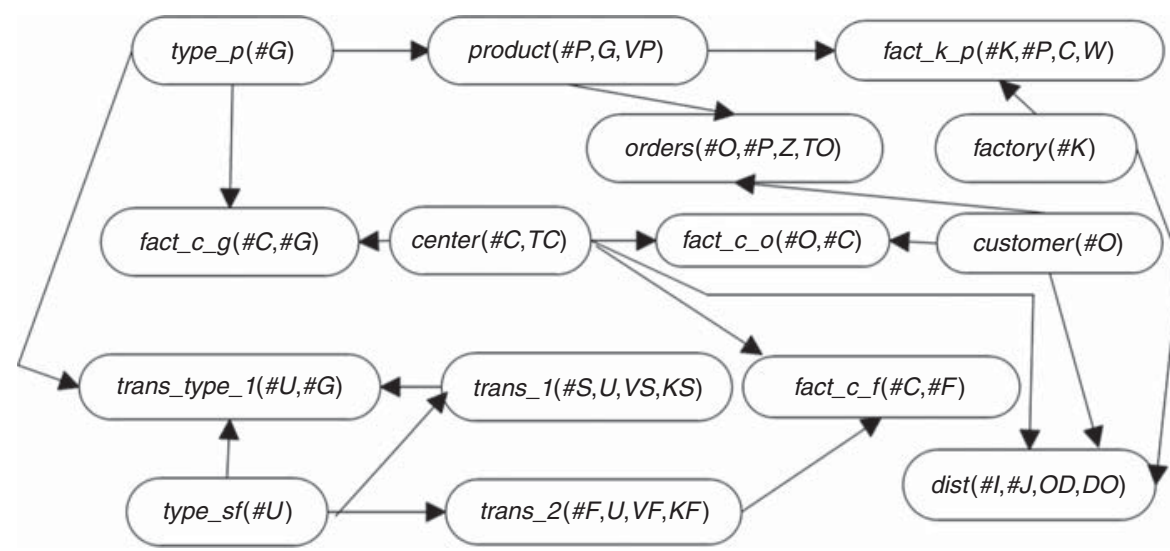

Note: \# - key attributes of the fact

Number Questions

Q1 What is the minimum cost of executing customer orders (including production and distribution costs)?

Q2 What is the minimum cost of executing customer orders (including only production costs)?

Q3 What is the minimum cost of executing customer orders if the selected means of transport units $S$ cannot participate in the distribution process?

Q4 What is the minimum cost of executing customer orders if the selected means of transport $F$ cannot participate in the distribution process?

Q5 What will the cost be of executing customers' orders if selected center $c$ is able to handle additional types of products?

Q6 How will the cost of executing customer orders change when the capacity of means of transport is decreased by $n \%$ ?

Q7 What is the minimum cost of executing customer orders if the selected means of transport $S$ and $F$ cannot participate in the distribution process?

\section{Table II.}

\section{The set of} management question in the constraintdriven approach
Q8 Is it possible to execution customer orders if no means of transport will be on the route longer than $T$ ?

Q9 Is it possible to execution customer orders to use no more than $K$ means of transport units?

Q10 Is it possible to execute customer orders if means of transport type $U$ will not transport product type $G_{i}$ and $G_{j}$ together?

is necessary to use a declarative approach. Answers to these questions are decisions determining whether the manner of distribution and logistics for the FSC meets certain conditions and constraints.

\subsection{Illustrative example - presolving}

The possibility of using a presolving technique is an important element of the proposed constraint-driven approach. Moreover, due to the structure of the model, presolving can be employed for each CSP and jointly for all CSPs. Presolving uses data-driven computing implemented in CLP. The general procedure is as follows. Some of the decision variables are eliminated based on specific instances of facts through the incorporated mechanisms: constraint propagation and dedicated predicates. Values for other variables are determined, some other variables are aggregated and transformed. This process results in the transformation of the constraint set of the model. Presolving gives the CLP ${ }^{\mathrm{T}}$-transformed 
model that includes noticeably fewer decision variables (often with a smaller number of indices) and a reduced set of constraints. This model has a smaller size and allows for finding a solution within a smaller solution space and within a considerably reduced search time.

The presolving method will be presented step-by-step for the model of the illustrative example.

In the first step, the subset of products, which is taken into account in further solving the model, is determined on the basis of the particular instance of the fact \#orders(\#O, \#P, Z, TO) (subset of the only products that customers ordered). For $\mathrm{CSP}_{1}$ (the problem of production and delivery from the factory to the distribution center), presolving includes decision variables $X_{k, p, c, o, s}$ and $Y_{k, c, s}$. It consists of the following steps:

- The lack of instances for specific values of attributes $k, p$ in the fact fact $k \_$(\#K, \#P, $C, W$ ) means that product $p$ cannot be produced at factory $k$. Thus, the corresponding indices of decision variables are not feasible/acceptable and de-activate suitable decision variables $X_{k, p, c, o, s}$.

- The combinations of attribute values $p, c$, which correspond to the situation in which the product type $g$ cannot be distributed by distribution center $c$, are determined on the basis of instances of facts fact_c_g $(\# C, \# G)$, product(\#P, $G, V P)$.

- The combinations of attribute values $p, s$, which correspond to the situation in which product type $g$ cannot travel using modes of transport $s$, are determined on the basis of instances of facts product(\#P, G, VP), type_sf(\#U), trans_1(\#S, $U, V S, K S)$, trans_type_1(\#U, \#G).

- The lack of instances for specific values of attributes $c, o$ in the fact fact_c_o(\#O, \#C) means that the given customer $c$ cannot be supplied from center $o$ (e.g. contracts, technical condition, etc.). Thus the corresponding indices of decision variables are not feasible/acceptable and de-activate suitable decision variables $X_{k, p, c, o, s}$.

- On the basis of the determined values of the decision variables $X_{k, p, c, o, s}$, some of the decision variables $Y_{k, c, s}$ are determined; the remaining variables are set to zero.

For $\mathrm{CSP}_{2}$ (the palletizing problem and preparation of shipping to customers), presolving includes aggregation of the decision variable $U o_{c, o, g}$ to $U_{h}$, defining the size of the pallet/ shipment containing product type $g$ delivered to the customer $o$ from distribution center $c$. It consists of the following steps:

- Existing combinations for values of attributes $c, o, g$ for the variable $U o_{c, o, g}$ are determined from variable $X_{k, p, c, o, s}$ and the instances of fact product( $\left.\# P, G, V P\right)$. Then the combinations are renumbered and assigned to a new attribute $h$. This is how new the variable, $U_{h}$, is created.

For $\mathrm{CSP}_{3}$ (the problem of delivering pallets/shipments from distributors to customers), presolving includes variables: $X H_{f, i, j}, Y H_{f, i, j, h}$ and $F H_{h, f}$. It consists of the following steps:

- The facts of existence are generated, only for existing attributes. These are $D O G_{h, g}$ (what type of products $g$ are included in shipment $h$ ), $D O S_{h, o}$, (to which customer $o$ shipment $h$ is delivered), $D O C_{h, c}$ (from which distribution center $c$ shipment $h$ is sent) and $D O F_{h, f}$ (can mode of transport $f$ carry shipment $h$ ).

- Based on decision variable $U_{h}$ and factors $D O G_{h, g}, D O F_{h, f}, D O S_{h, o}, D O C_{h, c}$ and facts

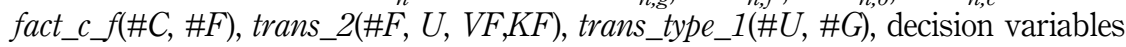
$Y_{f, i, j, h}$ are determined.

- On the basis of the determined values of the decision variables $Y_{f, i, j, h}$, decision variables $X_{f, i j}$ are determined; the remaining variables are set to zero. 
IMDS

117,9

2126

\subsection{Illustrative example - computational experiments}

In order to verify and evaluate the proposed constraint-driven approach, many computational experiments were performed for the illustrative example. All the experiments relate to the FSC with five manufacturers (factories) $(k=1, \ldots, 5)$, three distributor centers $(c=1, \ldots, 3)$, ten customers $(o=1, \ldots, 10)$, eight modes (types) of transportation $(u=1, \ldots, 8)$ and, 20 products $(p=1, \ldots, 20)$ which belong to the four product types (category) $(g=1, \ldots, 4)$. Numeric data for experiments (Appendix 2) were generated based on the actual distribution centers in the FMCG sector, which operate in the region of Central and Eastern Poland.

The experiments were carried out in two directions. First, we present how to apply the proposed approach to the illustrative model for all management questions Q1-Q10, showing its flexibility and decision support. Second, we present an analysis of the effectiveness of the proposed approach in the context of computing time and size of the problems solved.

For a comparative analysis, the same illustrative example for Q1 (question for the largest computational effort) has been implemented in two environments: MP and using a constraint-driven approach. In this case, the experiments were made for data instances which differed in the number of customer orders (from 1 to 200). All experiments were performed using a PC with the following parameters - Intel core (TM2), $2.6 \mathrm{GHz}, 4 \mathrm{~GB}$ of RAM. The results obtained for the first part of the experiments are shown in Table III.

Most of the questions have been parameterized, which increases the range of supported decision. As can be seen, the decision are related to the minimization of the cost of delivery for different conditions (Q1-Q7), for example, if one has a smaller number of means of transport (Q6A, Q6B), or inability to use certain means of transport (Q3, Q4, Q7). Other questions are related to the acceptable decisions (Q8-Q10). The answer to the question determines the value of all decision variables. Therefore, one can determine the method of delivery, the demand for transport, SC network, etc. Figures 7-10, respectively, show SC networks as a result of responses to questions Q1, Q7, Q5 and Q10.

The second part of the experiments yielded some very interesting findings. The results are shown in Table IV. As can be seen, the use of the constraint-driven approach has enabled finding a solution depending on the instance data up to 1,000 times faster than using the MP approach alone. For exact methods, this is a significant achievement. One can also note the reductions in the size of the model which is the result of the presolving technique. For example, the reduction of decision variables ranged from 36 to 1,000 times, and the reduction of constraints ranged from from 15 to 67 times.

\begin{tabular}{lccc}
\hline Question & Parameters & Answer & Objective-Fc \\
\hline Q1 & - & $\mathrm{Fc}$ & 5,310 \\
Q2 & - & $\mathrm{Fc}$ & 6,132 \\
Q3 & $S=\{\mathrm{s} 11, \mathrm{~s} 12\}$ & $\mathrm{Fc}$ & 5,430 \\
Q4 & $F=\{\mathrm{f} 01\}$ & $\mathrm{Fc}$ & 6,210 \\
Q5 & $C=\{\mathrm{c} 02\}, G=\{\mathrm{g} 03, \mathrm{~g} 04\}$ & $\mathrm{Fc}$ & 4,460 \\
Q6A & $n=30 \%$ & $\mathrm{Fc}$ & 5,390 \\
Q6B & $n=50 \%$ & $\mathrm{Fc}$ & 5,490 \\
Q7 & $S=\{\mathrm{s} 11, \mathrm{~s} 12\}, F=\{\mathrm{f} 01\}$ & $\mathrm{Fc}$ & 6,210 \\
Q8A & $T=45$ & YES & $5,310^{\mathrm{a}}$ \\
Q8B & $T=30$ & YES & $5,710^{\mathrm{a}}$ \\
Q8C & $T=20$ & NO & - \\
Q9A & $K=11$ & YES & $5,310^{\mathrm{a}}$ \\
Q9B & $K=10$ & NO & - \\
Q10 & $U=\{\mathrm{u} 01\}, G=\{\mathrm{g} 01, \mathrm{~g} 03\}$ & $\mathrm{Fc}$ & $6,290^{\mathrm{a}}$
\end{tabular}

Table III.

Result of the computational experiments answers for questions Q1-Q10

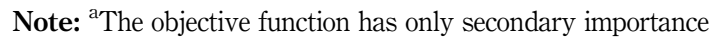




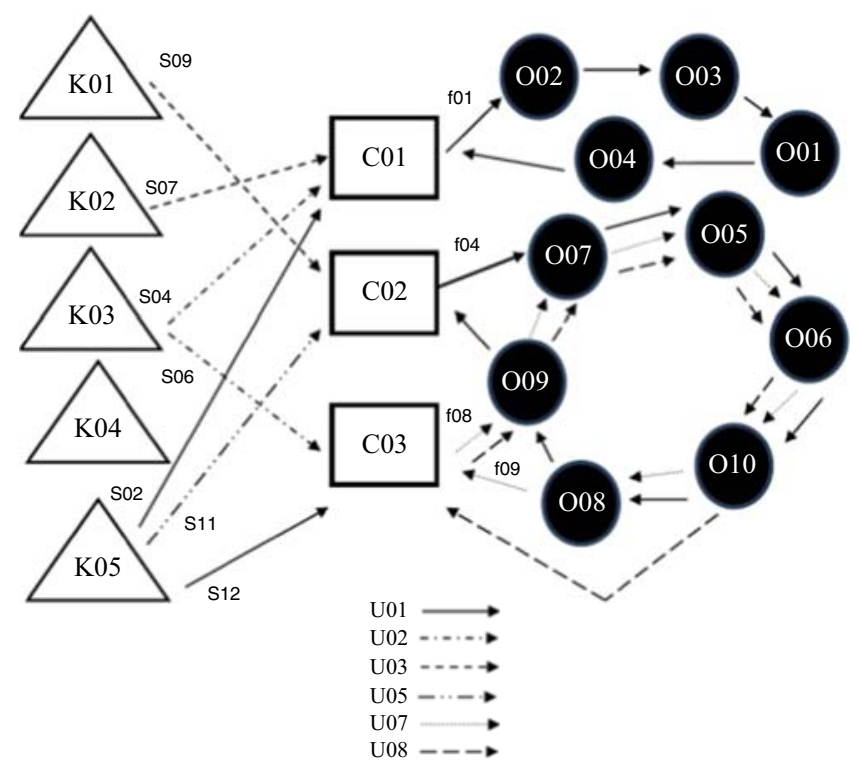

Food

supply chain management

Figure 7. The food supply chain network corresponding to the answer to the question Q1

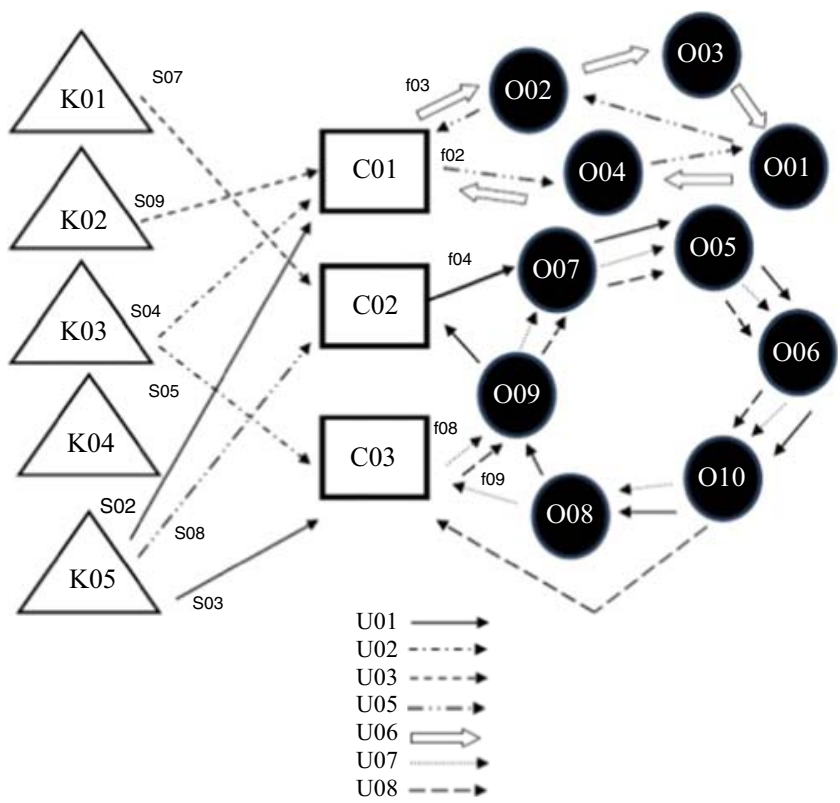

Figure 8. The food supply chain network corresponding to the answer to the question Q7

The main practical conclusions arising from the research results are as follows:

- The scope of decision support for FSCM is very wide and depends on the proposed set of questions that may be general or specific with additional logical conditions (Table III). Modeling decisions, as a response to questions, is natural and easy to use by practitioners: managers, decision makers, executive directors, etc. 
IMDS

117,9

2128

Figure 9.

The food supply chain network corresponding to the answer to the question Q5

Figure 10.

The food supply chain network corresponding to the answer to the question Q10
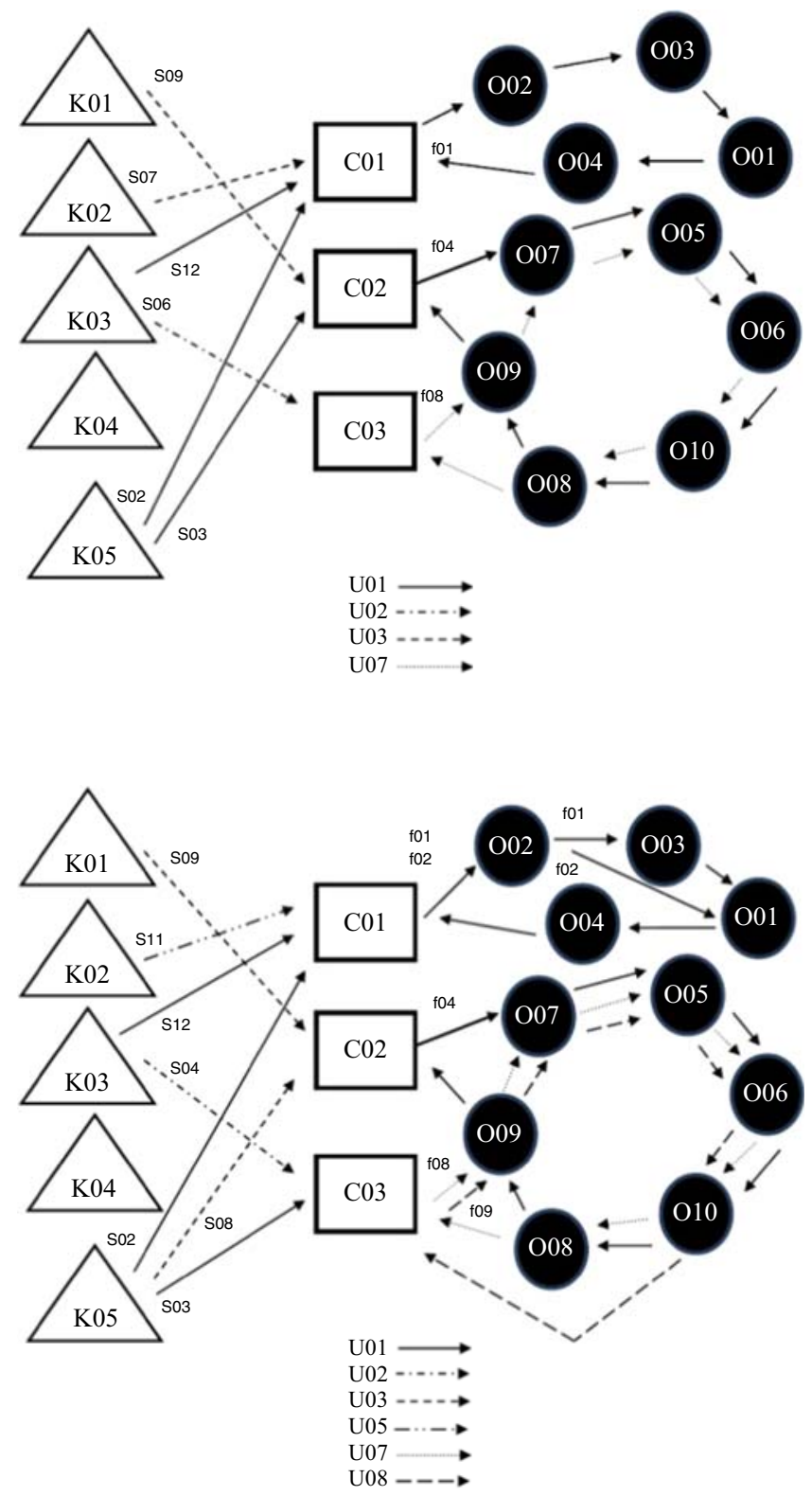

- The application of the constraint-driven approach to solving the proposed decisionmaking model is much more efficient than other exact methods currently used for this type of problem (Table IV).

- The constraint-driven approach can provide a framework for building dedicated decision support systems for FSCM. 


\begin{tabular}{lrrrrrrrr}
\hline & \multicolumn{3}{c}{ MP } & \multicolumn{4}{c}{ Constraint-driven approach } \\
Example & \multicolumn{1}{c}{ Fc } & \multicolumn{1}{c}{$T$} & \multicolumn{1}{c}{$C$} & Fc & $T$ & \multicolumn{1}{c}{$T$} & \multicolumn{1}{c}{$C$} \\
\hline $\mathrm{N}(1)$ & 706 & 94 & 875,678 & 56,756 & 706 & 3 & 688 & 845 \\
$N(15)$ & 819 & 210 & 875,678 & 78,456 & 819 & 3 & 1,717 & 903 \\
$N(50)$ & 4,979 & 556 & 875,678 & 94,565 & 4,979 & 3 & 9,601 & 8,745 \\
$N(67)$ & 5,310 & 886 & 875,678 & 123,654 & 5,310 & 4 & 11,788 & 9,756 \\
$N(80)$ & 5,632 & 996 & 875,678 & 145,675 & 5,632 & 5 & 13,227 & 9,821 \\
$N(100)$ & 5,696 & 1,276 & 875,678 & 148,456 & 5,696 & 4 & 15,535 & 9,886 \\
$N(125)$ & 6,309 & 1,456 & 875,678 & 148,756 & 6,309 & 7 & 17,814 & 9,888 \\
$N(150)$ & $7,098^{\mathrm{a}}$ & $1,500^{\mathrm{b}}$ & 875,678 & 149,567 & 7,098 & 6 & 19,920 & 9,892 \\
$N(200)$ & $8,456^{\mathrm{a}}$ & $1,500^{\mathrm{b}}$ & 875,678 & 152,345 & 7,715 & 7 & 24,120 & 9,896
\end{tabular}

Notes: Fc: objective function; $T$ : time of finding solution (in seconds); $V$ : the number of decision variables;

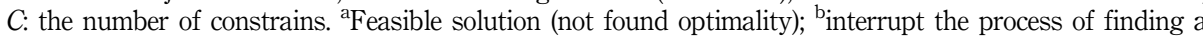
solution after a given time $1,500 \mathrm{~s}$

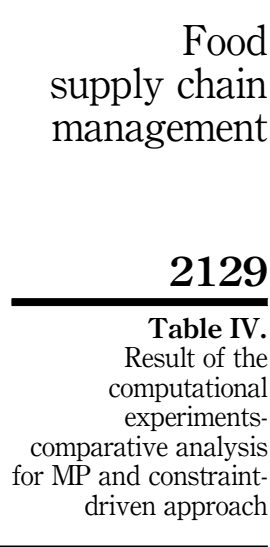

\section{Conclusions}

The main contributions of the research presented in the paper are:

- A new structure of the decision-making model (Figure 4) for FSCM is developed. The model applies to logistics and distribution in FSCs and consists of the sets of constraints, facts and questions.

- A specific question set that shapes individual decisions and the method for meeting the constraints (Table II) is presented.

- The model is implemented and solved using the authors' constraint-driven approach (Figure 3),

- The presolving method is applied and a detailed description is given (Section 4.3)

- A universal data structure in the form of a set of facts for the model is proposed and presented (Figure 6).

The proposed constraint-driven approach enables hybridization and presolving. Both of these features complemented by the declarativeness property allows building models of the structure, as in Figure 4, and makes this approach extremely flexible and effective. This flexibility enables modeling problems of varying structures and different types of constraints. This feature is particularly important in FSCM problems, where not only linear but also non-linear and logical constraints occur. Hybridization of CLP with environments other than the MP is also possible, e.g. with metaheuristics like (genetic algorithms), (ant colony optimization), (simulated annealing), etc. The effectiveness of the presented approach results from the application of presolving techniques based on data-driven computing and CLP-embedded methods.

The obtained results fully confirm both the flexibility and efficiency of the proposed approach (Section 4.4). Worthy of note is the wide range of supported decision for FSCM (Q1-Q10). Also, the results of performance of the proposed approach in terms of computation time and reduction in model size in relation to the application of the MP approach are very promising. Another objective is to compare the two approaches (constraint driven and MP) to verify the correctness of the results obtained by the constrained-driven approach. Further work on the development of the constraint-driven approach to FSCM will be carried out in different directions. One focus will be on expanding the set of questions. It is planned to extend the models with additional CSPs associated with traceability, temperature evolution during transportation, recycling and environmental issues, etc. It is also planned to integrate the CLP with different metaheuristics within the proposed constraint-driven approach. Replacing MP with certain metaheuristics in the proposed approach allows finding solutions to even larger size problems. 
IMDS

117,9

Ahumada, O. and Villalobos, J.R. (2009), "Application of planning models in the agri-food supply chain: a review”, European Journal of Operational Research, Vol. 196 No. 1, pp. 1-20.

Akkerman, R., Farahani, P. and Grunow, M. (2010), "Quality, safety and sustainability in food distribution: a review of quantitative operations management approaches and challenges", $O R$ Spectrum, Vol. 32 No. 4, pp. 863-904, available at: https://doi.org/10.1007/s00291-010-0223-2

Apt, K. and Wallace, M. (2006), Constraint Logic Programming using Eclipse, Cambridge University Press, Cambridge.

Banaeian, N., Mobli, H., Nielsen, I.E. and Omid, M. (2015), "Criteria definition and approaches in green supplier selection - a case study for raw material and packaging of food industry", Production and Manufacturing Research, Vol. 3 No. 1, pp. 149-168, available at: http://dx.doi.org/10.1080/2 1693277.2015.1016632

Banaeian, N., Mobli, H., Fahimnia, B., Nielsen, I.E. and Omid, M. (in press), "Green supplier selection using fuzzy group decision making methods: a case study from the agri-food industry", Computers and Operations Research, Vol. 90, doi: 10.1016/j.cor.2016.02.015.

Banaszak, Z.A., Zaremba, M.B. and Muszyński, W. (2009), "Constraint programming for project-driven manufacturing”, International Journal of Production Economics, Vol. 120 No. 2, pp. 463-475.

Bocewicz, G., Nielsen, I. and Banaszak, Z.A. (2016), "Production flows scheduling subject to fuzzy processing time constraints", International Journal of Computer Integrated Manufacturing, Vol. 29 No. 10, pp. 1105-1127, doi: 10.1080/0951192X.2016.1145739.

Bockmayr, A. and Kasper, T. (2004), "Branch-and-Infer: A framework for combining CP and IP", in Milano, M. (Ed.), Constraint and Integer Programming Operations Research/Computer Science Interfaces Series, Springer, Boston, MA, Vol. 27 No. 1, pp. 59-87, available at: https://doi.org/ 10.1007/978-1-4419-8917-8_3

Brown, G., Keegan, J., Vigus, B. and Wood, K. (2001), “The Kellogg company optimizes production, inventory, and distribution”, Interfaces, Vol. 31 No. 6, pp. 1-15.

Kang, K., Kang, C. and Hong, Y.S. (2014), "Data-driven optimized vehicle-level engineering specifications", Industrial Management \& Data Systems, Vol. 114 No. 3, pp. 338-364.

Kelepouris, T., Pramatari, K. and Doukidis, G. (2007), "RFID-enabled traceability in the food supply chain", Industrial Management \& Data Systems, Vol. 107 No. 2, pp. 183-200.

Kepler, E.F. (2004), "Supply chain approach to sustainable beef production from a Brazilian perspective”, Livestock Production Science, Vol. 90 No. 1, pp. 53-61.

Kutz, J.N. (2013), Data-Driven Modeling \& Scientific Computation: Methods for Complex Systems \& Big Data, Oxford University Press, Oxford.

Linton, J., Klassen, R. and Jayaraman, V. (2007), "Sustainable supply chains: an introduction, in", Journal of Operations Management, Vol. 25 No. 6, pp. 1075-1082, doi: 10.1016/j.jom.2007.01.012.

Milano, M. and Wallace, M. (2010), "Integrating operations research", Constraint Programming, Annals of Operations Research, Vol. 175 No. 1, pp. 37-76.

Nakandala, D., Lau, H. and Zhang, J. (2016), "Cost-optimization modelling for fresh food quality and transportation”, Industrial Management \& Data Systems, Vol. 116 No. 3, pp. 564-583.

Osvald, A. and Stirn, L.Z. (2008), "A vehicle routing algorithm for the distribution of fresh vegetables and similar perishable food", Journal of Food Engineering, Vol. 85 No. 2, pp. 285-295.

Power, D.J. (2008), "Understanding data-driven decision support systems", Information Systems Management, Vol. 25 No. 2, pp. 149-154. 
Rossi, F., Van Beek, P. and Walsh, T. (2006), Handbook of Constraint Programming (Foundations of Artificial Intelligence), Elsevier Science Inc., New York, NY.

Saha, S., Nielsen, I. and Moon, I. (2017), "Optimal retailer investments in green operations and preservation technology for deteriorating items", Journal of Cleaner Production, Vol. 140 No. 3, pp. 1514-1527, available at: https://doi.org/10.1007/978-1-4419-8917-8_3

Schimpf, J. and Kish, S. (2012), "ECLiPSe - from LP to CLP", Theory and Practice of Logic Programming, Vol. 12 Nos 1-2, pp. 127-156, doi: 10.1017/S1471068411000469.

Schrijver, A. (1998), Theory of Linear and Integer Programming, John Wiley \& Sons, New York, NY.

Sitek, P. (2014), "A hybrid CP/MP approach to supply chain modelling, optimization and analysis", Proceedings of the 2014 Federated Conference on Computer Science and Information Systems, pp. 1345-1352, doi:10.15439/2014F89.

Sitek, P. and Wikarek, J. (2013), "A hybrid method for modeling and solving constrained search problems", Federated Conference on Computer Science and Information Systems (FedCSIS 2013), pp. 385-392.

Sitek, P. and Wikarek, J. (2015), "A hybrid approach to the optimization of multiechelon systems", Mathematical Problems in Engineering, Vol. 2015, Article ID 925675, available at: http://dx.doi. org/10.1155/2015/925675

Sitek, P. and Wikarek, J. (2016), "A hybrid programming framework for modeling and solving constraint satisfaction and optimization problems”, Scientific Programming, Vol. 2016 No. 2016, Article ID 5102616, available at: http://dx.doi.org/10.1155/2016/5102616

Soto-Silva, W.E., Nadal-Roig, E., González-Araya, M.C. and Pla-Aragones, L.M. (2016), "Operational research models applied to the fresh fruit supply chain”, European Journal of Operational Research, Vol. 251 No. 2, pp. 345-355.

Soysal, M., Bloemhof-Ruwaard, J.M., Meuwissen, M.P.M. and van der Vorst, J.G.A.J. (2012), “A review on quantitative models for sustainable food logistics management", International Journal on Food System Dynamics, Vol. 3 No. 2, pp. 136-155.

Trienekens, J. and Zuurbier, P. (2008), "Quality and safety standards in the food industry, developments and challenges", International Journal of Production Economics, Vol. 113 No. 1, pp. 107-122, available at: https://doi.org/10.1016/j.ijpe.2007.02.050

Van der Vorst, J.G.A.J., Beulens, A.J.M and van Beek, P. (2005), "Innovations in logistics and ICT in food supply chain networks", in Jongen, W.M.F. and Meulenberg, M.T.G. (Eds), Innovation in Agrifood Systems: Product Quality and Consumer Acceptance, Wageningen Academic Publishers, Wageningen, pp. 245-292.

Wang, X., O'Brien, C. and Li, Y. (2010), "A production planning model to reduce risk and improve operations management", International Journal of Production Economics, Vol. 124 No. 2, pp. 463-474.

(The Appendix follows overleaf.) 
Values calculated

$M L_{p, s}$

$M C_{p, c}$

$S T$

Decision variables

$X_{k, p, c, o, s}$

$Y_{k, c, s}$
If the product $p$ can be delivered by means of transport $s$, then $M L_{p, s}=1$ otherwise $M L_{p, s}=0$

If the product $p$ can be operated by distribution center $c$, then $M C_{p, c}=1$ otherwise $M C_{p, c}=0$

The large fixed value, for example, the total number of ordered products

The size of product $p$ delivery from the manufacturer (factory) $k$ to the distribution center $c$ using means of transport $s$ due to the customer order $o$ If the delivery is carried out from manufacturer (factory) $k$ to the distribution center $c$ using means of transport $s$, then $Y_{k, c, s}=1$ otherwise $Y_{k, c, s}=0$

Constraints

The volume of supply to distribution centers from producers resulting from customer orders $\sum_{k \in K} \sum_{c \in C} \sum_{s \in S} B_{c, o} \cdot M L_{p, s} \cdot M C_{p, c} \cdot X_{k, p, c, o, s} \leqslant Z_{o, p} \forall 0 \in O, p \in P$

The manufacturer can not dispatch more products than its production capacity

$\sum_{c \in C} \sum_{o \in O} \sum_{s \in S} X_{k, p, c, o, s} \leqslant C K_{k, p} \forall k \in K, p \in P$

If the delivery is carried out from the factory to the distribution center, there must be a course specific means of transport (link decision variables $X_{k, p, c, o, s}$ and $Y_{k, c, s}$ )

$X_{k, p, c, o, s} \leqslant \mathrm{ST} \cdot Y_{k, c, s} \forall k \in K, p \in P, c \in C, o \in O, s \in S$

$\sum_{o \in O} \sum_{p \in P} X_{k, p, c, o, s} \geqslant Y_{k, c, s} \forall k \in K, c \in C, s \in S$

At most one course in a given period for a given means of transport

$\sum_{k \in K} \sum_{c \in C} Y_{k, c, s} \leqslant 1 \forall s \in S$

The volume (tonnage) loaded on the means of transport can not be greater than its capacity (tonnage) - note that this version of the model assumes only one gauge if it were to be considered that more similar equations arise only with other factors

$\sum_{o \in O} \sum_{p \in P} V P_{p} \cdot X_{k, p, c, o, s} \geqslant V F_{s} \cdot Y_{k, c, \forall} \forall k \in K, c \in C, s \in S$

Binarity and integrity

Table AI.
Indices, parameters,

decision variables and

$X_{k, p, c, o, s} \in Z^{+} \forall k \in K, p \in P, c \in C, o \in O, s \in S$ constrains for $\mathrm{CSP}_{1}$ 


\begin{tabular}{|c|c|}
\hline Symbol & Description \\
\hline \multicolumn{2}{|l|}{ Indices } \\
\hline$H$ & Set of pallets/grouped shipments \\
\hline$h$ & Shipment/pallet $(h=1, \ldots, H)$ \\
\hline \multicolumn{2}{|c|}{ Values calculated } \\
\hline$U_{h}$ & $\begin{array}{l}\text { The size of the shipment/pallet for aggregate index } h \\
\text { (based on } U O_{c, o, g} \text { ) }\end{array}$ \\
\hline$D O C_{h, c}$ & $\begin{array}{l}\text { If the shipment/pallet } h \text { is dispatched from the center } \\
c \text {, then } D O C_{h, c}=1 \text { otherwise } D O C_{h, c}=0\end{array}$ \\
\hline$D O G_{h g}$ & $\begin{array}{l}\text { If the shipment/pallet } h \text { contains product type } g \text {, then } \\
D O G_{h, g}=1 \text { otherwise } D O G_{h, g}=0\end{array}$ \\
\hline$D O F_{h, f}$ & $\begin{array}{l}\text { If the means of transport } f \text { can carry shipment } h \text {, then } \\
D O F_{h, f}=1 w \text { otherwise } D O F_{h, f}=0\end{array}$ \\
\hline \multicolumn{2}{|c|}{ Decision variables } \\
\hline$X_{k, p, c, o, s}$ & The same meaning as for the $\mathrm{CSP}_{1}$ \\
\hline$U O_{c, o, g}$ & $\begin{array}{l}\text { The size of the shipment/pallet for product type } g \\
\text { delivered to the customer } o \text { from the distribution } \\
\text { center } c\end{array}$ \\
\hline
\end{tabular}

\section{Food \\ supply chain management}

Constraints

Determination of the coefficient $M L_{p, s}$

$M L_{p, s}=\sum_{g \in G} \sum_{u \in U} A_{p, g} \cdot D_{u, g} \cdot H S_{s, u} \forall p \in P, s \in S$

Determination of the coefficient $M C_{p, c}$

$M C_{p, c}=\sum_{g \in G} A_{p, g} \cdot L_{c, g} \forall p \in P, c \in C$

Determination of the size of the shipment/pallet for product type $g$ delivered to the customer $o$ from the distribution center $c$

$\sum_{k \in K} \sum_{p \in P} \sum_{s \in S} V P_{p} \cdot A_{p, g} \cdot D_{u, g} \cdot H S_{s, u} X_{k, p, c, o, s}=U O_{c, o g} \forall c \in C, o \in O, g \in G$ (C9)

Aggregation and unification attributes the shipment/pallet and its renumbering

$h=[c, o, g] \forall c \in C, o \in O, g \in G: U O_{c, o, g}>0$

$U_{h}=U O_{c, o, g} \forall c \in C, o \in O, g \in G, u \in U: h=[c, o, g]$

$D O S_{h, o}=1 \forall c \in C, o \in O, g \in G, h \in H: h=[c, o, g]$

$D O C_{h, c}=1 \forall c \in C, o \in O, g \in G, h \in H: h=[c, o, g]$

Table AII.

Indices, parameters, decision variables and constrains for $\mathrm{CSP}_{2}$
Determination of the coefficient $D O F_{h, f}$

$D O F_{h, f}=1 \forall h \in H, f \in F, g \in G, u \in U: D O G_{h_{g} g}=1, D_{u, g}=1, H F_{f, u}=1$

Determination of the coefficient $D O G_{h, g}$
$D O G_{h, g}=1 \forall c \in C, o \in O, g \in G, h \in H, p \in P: h=[c, o, g], A_{p, g}=1$

constrains for $\mathrm{CSP}_{2}$


IMDS

117,9

\section{4}

Symbol

Decision variables

$X H_{f, i j}$

$Y H_{f, i, j, h}$

$F H_{h, f}$

\section{Description}

If the means of transport $f$ travels from point $i$ to point $j$, then $X_{f, i, j}=1$, otherwise $X_{f, i j}=0(f \in F, i \in O \cup C, j \in O \cup C)$

If the means of transport $f$ travels from point $i$ to point $j$ carrying shipment/pallet $h$, then $Y_{f, i, j, h}=1$, otherwise $Y_{f, i, j, h}=O(f \in F, i \in O \cup C$, $j \in O \cup C, h \in H$ )

If shipment/pallet $h$ is delivered by means of transport $f$ than $\mathrm{FH}_{h, f}=1$, otherwise $\mathrm{FH}_{h, f}=0(h \in H, f \in F)$

Constraints

Arrival and departure means of transport from the delivery point

$\sum_{j \in O \cup C} X H_{f, i, j}=\sum_{j \in O \cup C} X H_{f, j, i} \forall f \in F, i \in O \cup C$

If no shipments $h$ are to be carried on the route $i, j$, a means of transport does not travel that route - linking the decision variable $X H_{f, i, j}$ with decision variable $\mathrm{YH}_{f, i, h}$

$X H_{f, i, j} \leqslant \sum_{h \in H} Y H_{f, i, j, h} \forall f \in F, i \in O \cup C, j \in O$

$Y H_{f, i j, h} \leqslant X H_{f, i, j} \forall f \in F, i \in O \cup C, j \in O \cup C, h \in H$

The given means of transport carries no more shipments than allowable as per its capacity volume

$\sum_{h \in H} U_{h} \cdot Y H_{f, i j, h} \leqslant V F_{f} \forall f \in F, i \in O \cup C, j \in O \cup C$

Shipment being delivered to customer

$\sum_{f \in F} \sum_{i \in O \cup C} \mathrm{YH}_{f, i, j, h}=\operatorname{DOS}_{h, j} \forall h \in H, j \in O$

Means of transport $f$ carries at most one course/route in the planning period

$\sum_{j} X H_{f, c, j} \leqslant 1 \quad \forall f \in F, c \in C$

Means of transport $f$ carries the course/route from this distribution center to which it was assigned

$\sum_{j} X H_{f, c, j} \leqslant \mathrm{CF}_{c, f} \quad \forall f \in F, c \in C$

Items picked up/delivered to a delivery point

$\sum_{j \in O} \sum_{f \in F} Y H_{f, j, i, h}-\sum_{j \in O} \sum_{f \in F} Y H_{f, i, j, h}=D O S_{h, i} \forall i \in O, h \in H$

Shipment/pallet can be taken up only from the distribution center, where it is prepared

$\sum_{f \in F} \sum_{i \in O} \sum_{c \in C} D O C_{h, c} \cdot Y H_{f, c, i, h}=1 \quad \forall h \in H$

What means of transport the shipment is delivered to the customer

$Y H_{f, i, j, h}=F H_{h, f} \quad \forall f \in F, i \in O, j \in O, h \in H$

The given shipment/pallet is delivered by only one means of transport

$\sum_{f \in F} F H_{f, h}=1 \quad \forall h \in H$

A means of transport carrying only such shipment/pallet, which can carry because of the type of products in the shipment/pallet

Table AIII.

$F H_{h, f} \leqslant D O F_{h, f} \quad \forall h \in H, f \in F$

Binarity

$Y H_{f, i, j, h}=\{0,1\} \forall f \in F, i \in O \cup C, j \in O \cup C, h \in H$ 
Symbol

Description

Decision variables

$X_{k, p, c, o, s}$

$X O_{k, c, p, o, s}$

$X H_{f, i j}$

$T H_{f, o}$
The same meaning as for the $\mathrm{CSP}_{1}$ If product $p$ is delivered from the manufacturer (factory) $k$ to the distribution center $c$ using means of transport $s$, then $X O_{k, c, p, o, s}=1$, otherwise $X O_{k, c, p, o, s}=0$

The same meaning as for the $\mathrm{CSP}_{3}$ The total time of delivery to the customer $o$

Constrain

It determines the time of delivery, i.e. how long it takes for the delivery from the manufacturer (factory) to the customer

$$
\begin{aligned}
& X O_{k . p . c . o, s} \cdot S t \geqslant X_{k, p, c, o, s} \forall k \in K, c \in C, p \in P, o \in O, s \in S \\
& T H_{f, o}=\sum_{i \in O \cup C} \sum_{j \in O \cup C} D O_{i, j} \cdot X H_{f, i, j} \quad \forall f \in F, h \in H, o \in O: D O F_{h, f}=1 \\
& \sum_{k \in K} \sum_{c \in C} \sum_{s \in S}\left(D O_{k, c} \cdot X O_{k, p, c, o, s}+T C_{c} \cdot X O_{k, p, c, o, s}\right)+\sum_{h \in H} T H_{f, o}=T O_{p, o} \quad \forall p \in P, o \in O
\end{aligned}
$$

Table AIV.

Indices, parameters, decision variables and constrains for $\mathrm{CSP}_{\mathrm{TS}}$ 


\section{Appendix 2. Instances of facts for the illustrative example}

\#type_p(\#G) \#G-type of product (category)

type_p(g01). type_p (g02). type_p (g03). type_p(g04).

\#type_sf(\#U \#U - type of means of transport.

type_sf(u01). type_sf(u02). type_sf(u03). type_sf(u04). type_sf(u05).

type_sf(u06). type_sf(u07). type_sf(u08).

\#factory $(\# \mathbf{K}) \# \mathrm{~K}$ - manufacturer (factory)

factory (k01). factory (k02). factory(k03). factory(k04). factory(k05).

\#product(\#P,G,VP) \#P - product item, \#G - type of product, $\mathrm{VP}_{\mathrm{p}}-$ the volume/capacity occupied by product item

product $(p 01,2, g 01)$. product $(p 02,2, g 01)$. product $(p 03,3, g 01)$.

product $(\mathrm{p} 04,4, \mathrm{~g} 02)$. product $(\mathrm{p} 05,8, \mathrm{~g} 02)$. product $(\mathrm{p} 06,8, \mathrm{~g} 02)$.

product $(\mathrm{p} 07,7, \mathrm{~g} 03)$. product $(\mathrm{p} 08,9,903)$. product $(\mathrm{p} 09,6,903)$.

product $(\mathrm{p} 10,8, \mathrm{~g} 03)$. product $(\mathrm{p} 11,2, \mathrm{~g} 03)$. product $(\mathrm{p} 12,4, \mathrm{~g} 03)$.

product $(\mathrm{p} 13,7,903)$. product $(\mathrm{p} 14,9, \mathrm{~g} 03)$. product $(\mathrm{p} 15,3, \mathrm{~g} 03)$.

product $(\mathrm{p} 16,6, \mathrm{~g} 04)$. product $(\mathrm{p} 17,5, \mathrm{~g} 04)$. product $(\mathrm{p} 18,8, \mathrm{~g} 04)$.

product $(\mathrm{p} 19,5, \mathrm{~g} 04)$. product $(\mathrm{p} 20,7, \mathrm{~g} 04)$.

\#fact_k_p(\#K,\#P,C,W) \#K - manufacturer (factory), \#P - product item, $\mathrm{C}_{\mathrm{k}, \mathrm{p}}$ - production capacity at factory $\mathrm{k}$ for product $\mathrm{p}, \mathrm{W}_{\mathrm{k}, \mathrm{p}}$ - the production cost of product $\mathrm{p}$ at factory $\mathrm{k}$

fact $k p(k 1, p 01,100,1)$. fact $k p(k 1, p 02,100,2)$. fact $k p(k 1, p 01,100,4)$.

fact_k_p (k1,p04,100,8). fact_k_p (k1,p05,100,5). fact_k_p $(k 2, p 01,100,2)$.

fact_k_p $(k 2, p 02,100,3)$. fact_k_p $(k 2, p 01,100,5)$. fact_k_p $(k 2, p 04,100,6)$.

fact_k_p $(k 2, p 05,100,2)$. fact_k_p $(k 3, p 11,100,3)$. fact_k_p $(k 3, p 12,100,4)$.

fact_k_p $(k 3, p 13,100,1)$. fact_k_p $(k 3, p 14,100,2)$. fact_k_p $(k 3, p 15,100,4)$.

fact_k_p $(k 4, p 11,100,1)$. fact_k_p $(k 4, p 12,100,2)$. fact_k_p $(k 4, p 13,100,1)$.

fact $k p(k 4, p 14,100,3)$. fact $k p(k 4, p 15,100,6)$. fact $k p(k 5, p 06,100,2)$.

fact_k_p $(k 5, p 07,100,3)$. fact_k_p $(k 5, p 08,100,4)$. fact_k_p $(k 5, p 09,100,3)$.

fact_k_p $(k 5, p 10,100,3)$. fact_k_p $(k 5, p 16,100,1)$. fact_k_p $(k 5, p 17,100,3)$.

fact $k p(k 5, p 18,100,2)$. fact $k p(k 5, p 19,100,3)$. fact $k p(k 5, p 20,100,4)$.

\#center(\#C,TC) \#C - distribution center, $\mathrm{Tc}_{\mathrm{c}}$ - the time needed for distribution center $\mathrm{c}$ to prepare the shipment center $(\mathrm{c} 01,2)$. center $(\mathrm{c} 02,2)$. center $(\mathrm{c} 03,1)$.

\#customer(\#O) \#O - customers

customer(001). customer(002). customer(003). customer(004).

customer (005). customer(006). customer(007). customer(008).

customer(009). customer(010).

\#fact_c_o(\#O,\#C) \#O - customer, \#C - distribution center

fact_o_c $(001, \mathrm{c01})$. fact_o_c $(002, \mathrm{c01})$. fact_o_c $(003, \mathrm{c01})$.

fact_óc $(004, \mathrm{c} 01)$. fact_o_c $(005, \mathrm{c} 02)$. fact_o_c $(006, \mathrm{c02})$.

fact_o_c $(007, \mathrm{c02})$. fact_o_c $(008, \mathrm{c} 03)$. fact_o_c $(009, \mathrm{c} 02)$.

fact ${ }^{-} \mathrm{c}(010, \mathrm{c02})$. fact $\mathrm{O}^{-} \mathrm{c}(005, \mathrm{c03})$. fact $\mathrm{O}^{-} \mathrm{c}(006, \mathrm{c03})$.

fact_o_c $(007, c 03)$. fact_o_c $(008, c 03)$. fact_o_c $(009, c 03)$.

fact_o_c $(010, \mathrm{c03})$. fact_o_c $(009, \mathrm{c03})$. fact_o_c $(010, \mathrm{c03})$.

\#fact_c_g(\#C,\#G) \#C - distribution center, \#G - type of product (category)

fact_c_g (c01,g01). fact_c_g (c01,g02). fact_c_g $(c 01, g 03)$.

fact_c_g (c01,g02). fact_c_g(c01,g01). fact_c_g(c01,g02).

fact_c_g (c03,g03). fact_c_g (c03,g04).

\#orders(\#O,\#P,Z,TO) \#O - number of customers, \#P - product item, $\mathrm{Z}_{\mathrm{o}, \mathrm{p}}$ - customer demand/order for product item, $\mathrm{TO}_{\mathrm{o}, \mathrm{p}}-$ the cut-off time of delivery to the delivery point/customer of product item

orders $(001, \mathrm{p} 01,8,40)$. orders $(001, \mathrm{p} 02,4,40)$. orders $(001, \mathrm{p} 03,5,40)$.

$\operatorname{orders}(001, \mathrm{p} 04,6,40)$. orders $(001, \mathrm{p} 08,4,40)$. $\operatorname{orders}(001, \mathrm{p} 09,5,40)$.

$\operatorname{orders}(001, \mathrm{p} 11,7,40)$. orders $(001, \mathrm{p} 12,8,40)$. orders $(001, \mathrm{p} 13,5,40)$. 
$\operatorname{orders}(001, \mathrm{p} 14,4,40) . \operatorname{orders}(001, \mathrm{p} 16,3,40) . \operatorname{orders}(001, \mathrm{p} 17,3,40)$. $\operatorname{orders}(001, \mathrm{p} 18,4,40)$. orders $(001, \mathrm{p} 19,4,40)$. orders $(001, \mathrm{p} 20,6,40)$. $\operatorname{orders}(002, \mathrm{p} 02,4,40)$. orders $(002, \mathrm{p} 05,5,40)$. orders $(002, \mathrm{p} 06,6,40)$. $\operatorname{orders}(002, \mathrm{p} 07,7,40)$. $\operatorname{orders}(003, \mathrm{p} 16,4,30)$. $\operatorname{orders}(003, \mathrm{p} 17,4,30)$. $\operatorname{orders}(003, \mathrm{p} 18,5,30)$. orders $(003, \mathrm{p} 19,5,30)$. $\operatorname{orders}(003, \mathrm{p} 20,8,30)$. $\operatorname{orders}(004, \mathrm{p} 01,2,20)$. orders $(004, \mathrm{p} 02,2,20)$. orders $(004, \mathrm{p} 06,2,20)$. orders $(004, \mathrm{p} 07,2,20)$. orders $(004, \mathrm{p} 09,2,20)$. orders $(004, \mathrm{p} 13,2,20)$. $\operatorname{orders}(005, \mathrm{p} 03,3,40)$. orders $(005, \mathrm{p} 04,4,40)$. orders $(005, \mathrm{p} 06,5,40)$. $\operatorname{orders}(005, \mathrm{p} 07,6,40)$. orders $(005, \mathrm{p} 11,7,40)$. $\operatorname{orders}(005, \mathrm{p} 16,5,40)$. $\operatorname{orders}(005, \mathrm{p} 18,5,40)$. orders $(006, \mathrm{p} 01,4,40)$. orders $(006, \mathrm{p} 02,4,40)$. $\operatorname{orders}(006, \mathrm{p} 06,8,40)$. $\operatorname{orders}(006, \mathrm{p} 07,8,40) \cdot \operatorname{orders}(006, \mathrm{p} 11,4,40)$. $\operatorname{orders}(006, \mathrm{p} 12,4,40)$. $\operatorname{orders}(006, \mathrm{p} 16,7,40) . \operatorname{orders}(006, \mathrm{p} 20,8,40)$. $\operatorname{orders}(007, \mathrm{p} 01,2,50)$. orders $(007, \mathrm{p} 02,2,50)$. orders $(007, \mathrm{p} 03,2,50)$. $\operatorname{orders}(007, \mathrm{p} 04,2,50)$. orders $(007, \mathrm{p} 06,4,50)$. orders $(007, \mathrm{p} 07,4,50)$. $\operatorname{orders}(007, \mathrm{p} 13,5,50)$. orders $(007, \mathrm{p} 14,5,50)$. orders $(007, \mathrm{p} 16,6,50)$. $\operatorname{orders}(007, \mathrm{p} 18,7,50)$. $\operatorname{orders}(007, \mathrm{p} 20,8,50)$. $\operatorname{orders}(008, \mathrm{p} 01,1,40)$. $\operatorname{orders}(008, \mathrm{p} 02,2,40)$. $\operatorname{orders}(008, \mathrm{p} 06,5,40)$. $\operatorname{orders}(008, \mathrm{p} 10,7,40)$. $\operatorname{orders}(008, \mathrm{p} 11,8,40)$. orders $(009, \mathrm{p} 01,1,60)$. orders $(009, \mathrm{p} 03,3,60)$. $\operatorname{orders}(009, \mathrm{p} 07,5,60)$. orders $(009, \mathrm{p} 12,8,60)$. $\operatorname{orders}(009, \mathrm{p} 13,8,60)$. $\operatorname{orders}(009, \mathrm{p} 16,4,60)$. $\operatorname{orders}(009, \mathrm{p} 17,5,60) . \operatorname{orders}(010, \mathrm{p} 01,1,40)$. $\operatorname{orders}(010, \mathrm{p} 04,4,40)$. $\operatorname{orders}(010, \mathrm{p} 08,6,40)$. $\operatorname{orders}(010, \mathrm{p} 09,6,40)$. $\operatorname{orders}(010, \mathrm{p} 14,2,40)$. $\operatorname{orders}(010, \mathrm{p} 15,3,40) . \operatorname{orders}(010, \mathrm{p} 18,6,40)$. $\operatorname{orders}(010, \mathrm{p} 19,7,40)$. orders $(010, \mathrm{p} 20,8,40)$.

\section{Food supply chain management}

\#trans_1(\#S,U,VS,KS)\#S - means of transport unit (from factory to distribution center ), U - type of means of transport, $\mathrm{VS}_{\mathrm{s}}-$ the volume/capacity for means of transport unit $\mathrm{s}, \mathrm{KS}_{\mathrm{s}}$ - kilometer cost of means of transport unit s

$$
\begin{aligned}
& \text { trans_1(s01,u01,1500,70). trans_1(s02,u01,1500,50). } \\
& \text { trans_1(s03,u01,1500,50). trans_1 (s04,u02,1200,40). } \\
& \text { trans_1 }{ }^{-}(\mathrm{s} 05, \mathrm{u} 02,1200,40) \text {. trans_1 } 1(\mathrm{~s} 06, \mathrm{u} 02,900,40) \text {. } \\
& \text { trans_1 (s07,u03,900,30). trans_1 (s08,u03,900,30). } \\
& \text { trans_1(s09,u03,900,30). trans_1(s10,u04,900,03). } \\
& \text { trans_1 (s11,u05,900,30). trans_1(s12,u01,900,30). }
\end{aligned}
$$

\#trans_2(\#F,U,VF, KF) \#F - means of transport unit (from distribution center to customer), $\mathrm{U}$ - type of means of transport, $\mathrm{VF}_{\mathrm{f}}-$ the volume/capacity for means of transport unit $\mathrm{f}, \mathrm{KF}_{\mathrm{f}}-$ kilometer cost of means of transport unit $\mathrm{f}$

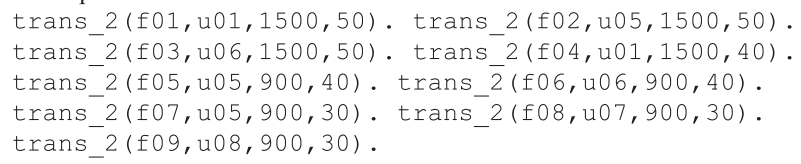

\#trans_type_1(\#U,\#G) \#U - type of means of transport, \#G - type of product (category)

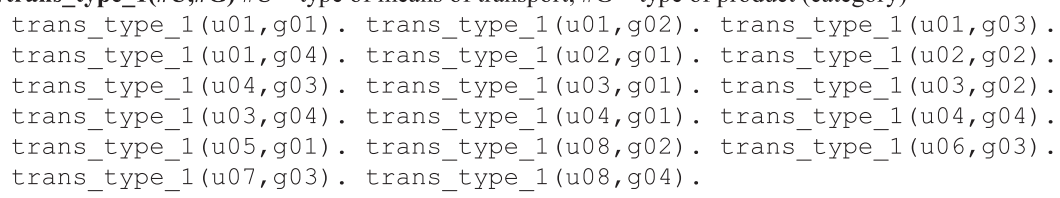

\#fact_c_f( $\mathbf{A C}$,\#F) \#C - distribution center, \#F - means of transport unit

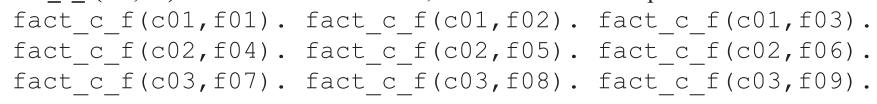


\#dist(\#I,\#J,OD,DO) \#I - start point: factory or distribution center or customer, \#J - end point: factory or distribution center or customer, $\mathrm{OD}_{\mathrm{i}, \mathrm{j}}$ - the distance between points, $\mathrm{DO}_{\mathrm{i}, \mathrm{j}}$ - travel time between points

dist $(f 01, \mathrm{c} 01,4,7)$. dist $(\mathrm{f} 02, \mathrm{c} 01,3,5)$. dist $(\mathrm{f} 03, \mathrm{c01}, 4,7)$. dist $(\mathrm{f} 04, \mathrm{C} 01,9,16)$ dist (f05, c01, 1,2). dist $(\mathrm{c} 01,001,6,11)$. dist $(\mathrm{c} 01,004,6,11)$. dist $(\mathrm{c} 01,007,5,10)$. dist $(\mathrm{c} 01,010,3,3)$. dist $(\mathrm{c} 02,003,4,6)$. dist $(\mathrm{c} 02,006,4,8)$. dist $(c 02,009,2,2)$. dist $(\mathrm{c} 03,002,3,5)$. dist $(\mathrm{c} 03,005,8,15)$. dist $(\mathrm{c} 03,008,6,6)$. dist $(001,002,7,14)$. dist $(001,005,4,6)$. dist $(001,008,6,6)$. dist $(002,001,7,14)$. dist $(002,005,6,10)$. dist $(002,008,3,3)$. dist $(003,001,7,13)$. dist $(003,005,7,14)$. dist $(003,008,6,6)$. dist $(004,001,3,5)$. dist $(004,005,2,3)$. dist $(004,008,5,5)$. dist $(005,001,4,6)$. dist $(005,004,2,3)$. dist $(005,008,3,3)$. dist $(006,001,4,7)$. dist $(006,004,4,6)$. dist $(006,008,2,2)$. dist $(007,001,2,3)$. dist $(007,004,5,9)$. dist $(007,008,6,5)$. dist $(008,001,6,6)$. dist $(008,004,5,5)$. dist $(008,007,6,5)$. dist $(009,001,5,5)$. dist $(009,004,8,8)$. dist $(009,007,3,3)$. dist $(010,001,8,7)$. dist $(010,004,6,6)$. dist $(010,007,8,7)$. dist ( $f 01, \mathrm{C02}, 4,7)$. dist (f02, c02,5,9). dist (f03, C02,4,7). dist (f04, C02,4,6). dist $(f 05, c 02,6,12)$ dist $(\mathrm{c} 01,002,2,3)$. dist $(\mathrm{c} 01,005,4,6)$. dist $(\mathrm{c} 01,008,1,1)$. dist $(\mathrm{c} 02,001,4,6)$. dist $(\mathrm{c} 02,004,6,11)$. dist $(c 02,007,1,2)$. dist $(\mathrm{c} 02,010,8,7)$. dist $(c 03,003,2,3)$. dist $(\mathrm{c} 03,006,7,12)$. dist $(c 03,009,5,5)$. dist $(001,003,7,13)$. dist $(001,006,4,7)$. dist $(001,009,5,5)$. dist $(002,003,4,6)$. dist $(002,006,4,7)$. dist $(002,009,6,5)$. dist $(003,002,4,6)$. dist $(003,006,6,11)$. dist $(003,009,3,3)$. dist $(004,002,8,15)$. dist $(004,006,4,6)$. dist $(004,009,8,8)$. dist $(005,002,6,10)$. dist $(005,006,1,2)$. dist $(005,009,7,7)$. dist $(006,002,4,7)$. dist $(006,005,1,2)$. dist $(006,009,6,6)$. dist $(007,002,6,11)$. dist $(007,005,4,8)$. dist $(007,009,3,3)$. dist $(008,002,3,3)$. dist $(008,005,3,3)$. dist $(008,009,7,3)$. dist $(009,002,6,5)$. dist $(009,005,7,7)$. dist $(009,008,7,3)$. dist $(010,002,5,5)$. dist $(010,005,4,4)$. dist $(010,008,2,1)$. dist ( $f 01, \mathrm{c03}, 8,15)$. dist ( $f 02, \mathrm{c03}, 2,3)$. dist ( $103, \mathrm{c} 03,1,2$ ). dist ( $\mathrm{f0} 4, \mathrm{c03}, 8,15)$. dist ( $\mathrm{f05}, \mathrm{c03}, 6,11)$. dist $(\mathrm{c} 01,003,5,9)$. dist $(c 01,006,2,3)$. dist $(\mathrm{c} 01,009,6,6)$. dist $(\mathrm{c02}, 002,5,9)$. dist $(c 02,005,5,9)$. dist $(\mathrm{c} 02,008,6,5)$. dist $(c 03,001,9,16)$. dist (c03,004,10,19). dist $(\mathrm{c03}, 007,7,12)$. dist $(\mathrm{c} 03,010,8,8)$. dist $(001,004,3,5)$. dist $(001,007,2,3)$. dist $(001,010,8,7)$. dist $(002,004,8,15)$. dist $(002,007,6,11)$. dist $(002,010,5,5)$. dist $(003,004,9,17)$. dist $(003,007,5,9)$. dist $(003,010,8,8)$. dist $(004,003,9,17)$. dist $(004,007,5,9)$. dist $(004,010,6,6)$. dist $(005,003,7,14)$. dist $(005,007,4,8)$. dist $(005,010,4,4)$. dist $(006,003,6,11)$. dist $(006,007,4,7)$. dist $(006,010,4,3)$. dist $(007,003,5,9)$. dist $(007,006,4,7)$. dist $(007,010,8,7)$. dist $(008,003,6,6)$. dist $(008,006,2,2)$. dist $(008,010,2,1)$. dist $(009,003,3,3)$. dist $(009,006,6,6)$. dist $(009,010,9,4)$. dist $(010,003,8,8)$. dist $(010,006,4,3)$. dist $(010,009,9,4)$.

\section{Corresponding author}

Pawel Sitek can be contacted at: sitek@tu.kielce.pl

For instructions on how to order reprints of this article, please visit our website: 